\title{
Improved Learning Scheme for Cognitive Radio using Artificial Neural Networks
}

\author{
Rita Mahajan, Deepak Bagai \\ Electronics and Communication Engineering Department, PEC University of Technology, Chandigarh, India
}

\begin{tabular}{l}
\hline Article Info \\
\hline Article history: \\
Received Sep 30, 2015 \\
Revised Nov 1, 2015 \\
Accepted Nov 18, 2015 \\
\hline
\end{tabular}

\section{Keyword:}

Artificial neural networks Cognitive radio networks Dynamic spectrum access Wireless communication

\begin{abstract}
The future of wireless system is facing the problem of spectrum scarcity. Number of users is increasing rapidly but available spectrum is limited. The Cognitive Radio (CR) network technology can enable the unlicensed users to share the frequency spectrum with the licensed users on a dynamic basis without creating any interference to primary user. Whenever secondary user finds that primary user is not transmitting and channel is free then it uses channel opportunistically. In this paper cognitive radio with predictive capability using artificial neural network has been proposed. The advantage of such cognitive user is saving of time and energy for spectrum sensing. Proposed radio will sense only that channel which is predicted to be free and channel is selected on the basis of maximum vacant time. Performance has been evaluated in the term of mean square error. The results show that this learning capability can be embedded in secondary users for better performance of future wireless technologies.
\end{abstract}

Copyright (C) 2016 Institute of Advanced Engineering and Science. All rights reserved.

\section{Corresponding Author:}

Rita Mahajan,

Departement of Electronics and Communication Engineering,

PEC University of Technology,

Sector 12, Chandigarh, India

Email: ritamahajan@pec.ac.in

\section{INTRODUCTION}

Modern advancements in wireless equipments lead to data transfer capacity of 1-10Mbps. In the upcoming times, this can be upgraded to nearly 100Mbps and then to $1 \mathrm{Gbps}$ in the next ten years. This capability to handle data at very high speed will enable users to easily handle high resolution images, high quality audio and video. However, it's often overlooked that as high performance wireless data services are widely deployed, deficiency of additional frequency spectrum will become a very serious limitation. The problem is, frequency allocation is fixed and is done by coordination between countries [1]. The problem of deficiency of spectrum can be observed from US Frequency Allocation chart [2]. Recent research on spectrum usage shows that the maximum usage is only $6 \%$ as shown in Figure 1 [3]. The inefficiency in the spectrum usage leads to a new technique to access wireless frequency spectrum opportunistically [4].

The underutilization of electromagnetic spectrum leads us to think in terms of spectrum holes, for which the following definition has been offered:

"A spectrum hole is a band of frequencies assigned to a primary user, but, at a particular time and specific geographic location, the band is not being utilized by that user". The inefficiency in the spectrum usage necessitates a new communication paradigm to exploit the existing wireless spectrum opportunistically [2-3]. Cognitive radio operation can be divided into three phases: radio-scene analysis, channel-state estimation and configuration selection. It can be considered as one of the intelligent frequency reuse scheme [5]. 


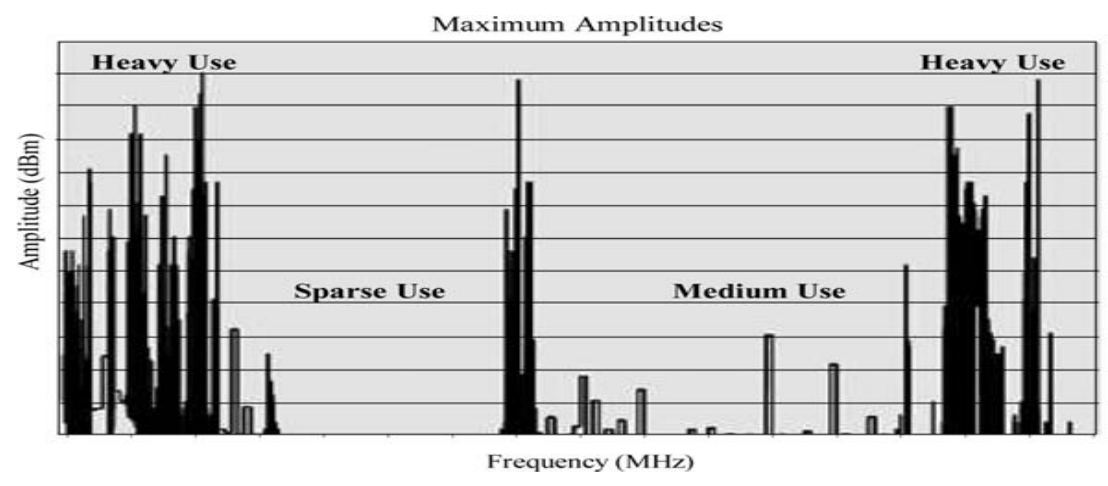

Figure 1. View of the actual availability of spectrum.

A frequency spectrum is assigned on payment basis to a number of Primary Users. Secondary Users can scan for unoccupied channels (spectrum holes) within the same spectrum band and communicate in that band. The way to detect holes in a spectrum is channel-by-channel scanning. [6]. CR can also be termed as software-controlled radio that can sense the environment and adjust its parameters accordingly on run time [7]. These additional cognitive capabilities in software defined radios are provided by the cognitive engine (CE) as shown in Figure 2. The CE helps the software-defined radio to adjust the parameters based upon the knowledge base.

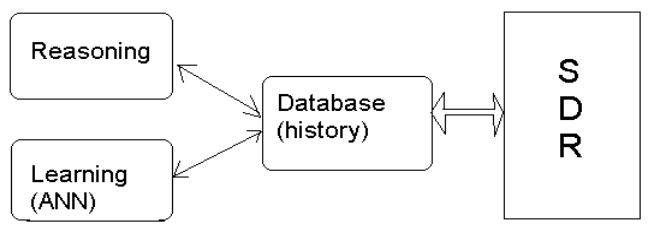

Figure 2. CR as software defined radio with intelligence

In this paper we propose a technique for inculcating the intelligence in cognitive radio using Artificial Neural Network. The ANN will predict the channels which will be vacant in future based on the previous history of channel states. It helps the cognitive user to sense only those channels and will save its time and energy.

The structure of the paper is as follows. In section II a review of Artificial Neural Network is presented and related work is projected in section III and the system model is discussed in Section IV, and the architecture and training procedure of the proposed network for is presented in Section V. The results of simulation in terms of the accuracy of the proposed learning scheme are discussed in Section VI. Then in the last section conclusion is presented.

\section{ARTIFICIAL NEURAL NETWORK}

An artificial neural network (ANN) consists of several processing units, called neurons. Within neural network three types of neurons are present (shown in Figure 3): input, hidden and output neurons. Each connection is defined by a weight, $\mathrm{w}_{\mathrm{jk}}$, which determine the effect that the signal of neuron $\mathrm{j}$ has on neuron k. Each neuron has a state of activation, be it $y_{k}$, called output of the neuron. During processing, each neuron $\mathrm{k}$ receives input $\mathrm{s}_{\mathrm{k}}$ from (a) neighbors belonging to different layers, and from (b) external sources called bias offset $b_{k}$, and uses them to compute an updated level of activation $y_{k}$. This is done by the use of an activation function $F_{k}$ as follows (shown in Figure 4) 


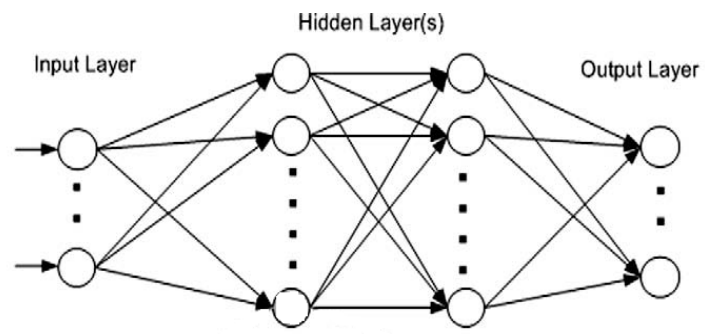

Figure 3. Typical neural network architecture

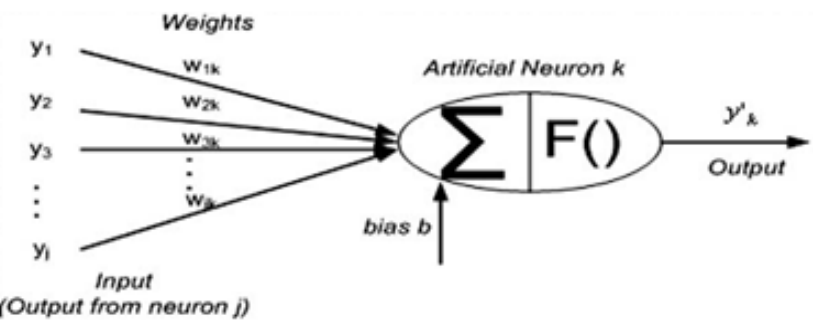

Figure 4. Neuron structure

Weighted sum of the inputs coming from the outputs of neurons in the previous layer is $S_{k}$ and is given by

$$
y_{k_{\_} \text {in }}=\sum_{i=1}^{n} w_{i k} \cdot x_{i}+b_{0 k}
$$

Expression for these transfer functions is as follows:
1. $\mathrm{y}_{\mathrm{k}}=\mathrm{F}_{\mathrm{k}}\left(y_{k_{-} \text {in }}\right)=y_{k_{-} i n}$
------ linear transfer function.
2. $\mathrm{y}_{\mathrm{k}}=\mathrm{F}_{\mathrm{k}}\left(y_{k_{-} i n}\right)=\frac{1}{1+e^{-y_{k_{-} i n}}}$
3. $\mathrm{y}_{\mathrm{k}}=\mathrm{F}_{\mathrm{k}}\left(y_{k_{-} i n}\right)=\frac{2}{1+e^{-y_{k_{-} i n}}}-1$
------ logistic-sigmoid.
------ hyperbolic tangent sigmoid.

In this paper, we use Multilayer Perceptron (MLP), a feedforward structure, which has been used widely in time series prediction and binary prediction. It is apparent that NN has to be trained such that the application of a set of inputs produces the desired set of outputs. This process is called learning or training and can be achieved by properly adjusting the weights $w_{j k}$ of the connections among all (j, $k$ ) neuron pairs. Training adjusts connection weights to produce the desired output which can be achieved by weight-changing procedure of the connection is termed as the backpropagation learning rule.

\section{RELATED WORK}

In literature [8-9] authors discusses the role of learning in CRs and emphasizes how crucial the independent learning ability in realizing a real CR device. They have presented a survey of the state-of the art achievements in applying machine learning techniques. They have also reviewed several CR implementations that are used as the various artificial intelligence techniques. It has been concluded in [8] that they do not provide any means of learning from past experiences, thus failing to exhibit one of the key properties of CR.

In [10-12] authors have proposed Elman Recurrent Neural Networks to predict the spectrum occupancy by modeling RF time series. In this paper [13], authors have design the channel status predictor using the neural network model multilayer perceptron (MLP). In cognitive radio networks, it is not possible to obtain the data of channel usage by the primary users. The advantage of neural networks is that it does not require a priori knowledge of the underlying distributions of the observed process. So, the neural networks are the good choice for modeling the channel status predictor. 
In [14] authors have analyzed various building blocks of DSA operation which can be incorporated into future LTE standards. They have also discussed the operational signaling scenarios to support cooperative sensing techniques, coordination and monitoring of frequency access rules. The authors in [15] utilize the framework presented in [14] and introduce the opportunistic spectrum access in LTE-A network. Their work illustrates the adoption of a geo-location database within LTE-A network that gathers the supportive information from the CR users with its neighboring environment.

Many researchers have applied various artificial neural networks in cognitive radio and achieved good results because of their special characteristics. Baldo and Zorzi [17] proposed a multilayered feedforward neural network for performance of real time communication for cognitive radio network. The function approximation capability of MFNN is used to performance characterization of the cognitive radio system. The cognitive user should be able to gather the relevant environment parameters which have significant impact on its performance.

In paper [18] authors have designed a neural network for decision making of Cognitive Engine, which is based on evaluation, and learning. This model of cognitive engine is compared with Reiser's model which is based on only unchangeable information [19]. An Adaptive Resonance Theory ART2 neural network has been proposed for spectrum sensing in paper [20]. This neural network satisfies the cognitive Wireless Mesh Network structure which combines with signal broadcast system.

\section{SYSTEM MODEL}

Our proposed system model for future wireless communication network (LTE- Advanced (5G)) consists of Dynamic spectrum access with additional capability of prediction for secondary users as shown in Figure 5. These secondary users are called cognitive users with prediction (CUP). Normal cognitive users (CU) sense all channels and find the spectrum hole for their use. The advantage of CUP over CU is that former senses only those channels which are predicted to be vacant and saves time and energy for spectrum sensing. CUP will select the vacant channel which has been free for longer time. This is done by additional equipment in normal CU called Artificial Neural Network. It will predict the vacant channel and its idle time based on the previous history. Therefore each CUP needs to maintain the database of status of the channels and this information can be used to train the ANN in control unit. The performance of this model will increase significantly over the existing model.

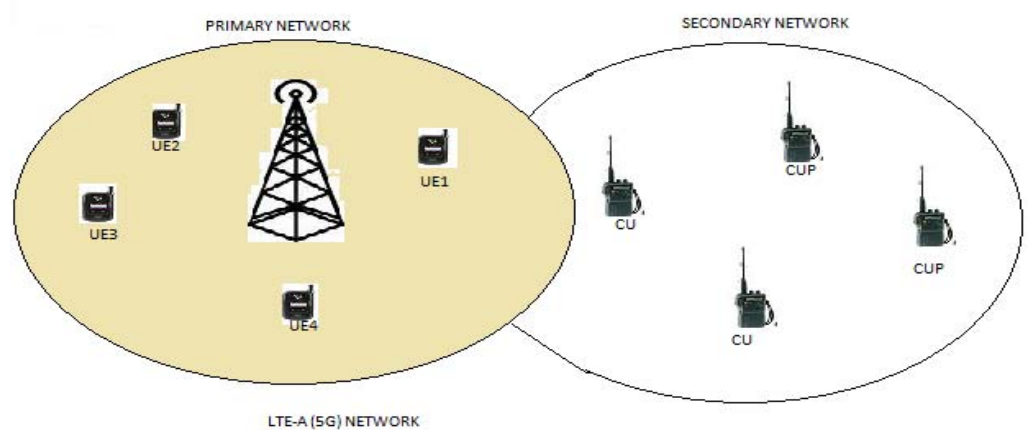

Figure 5. Scenario of future LTE-A wireless network

In LTE-A any user equipment can sense the spectrum in allotted sense time slot out of available time slot [16]. According to the standard, measurement gap length (MGL) is reserved for extracting the samples from a certain bandwidth and is fixed for 6 milliseconds. During the measurement gaps the user equipment shall not transmit any data and is not expected to tune its receiver on any carrier frequency. Another term measurement gap repetition period (MGRP) defined in the same standard as the time allotted to sense and transmission, and is fixed for 40 milliseconds shown in Figure 6. 


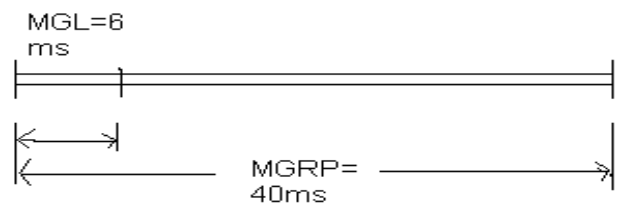

Figure 6. Measurement gap repetition period

\section{ARCHITECTURE AND TRAINING OF PREDICTOR}

The network used here is a multilayered structure which consists of an input layer, an output layer, and one or more hidden layers shown in Figure 7. The input layer has linear transfer function where as hidden and output layers have hyperbolic tangent sigmoid function (discussed in section II). The total number of inputs in the input layer is called the order of the MLP network and is denoted by $\tau$.

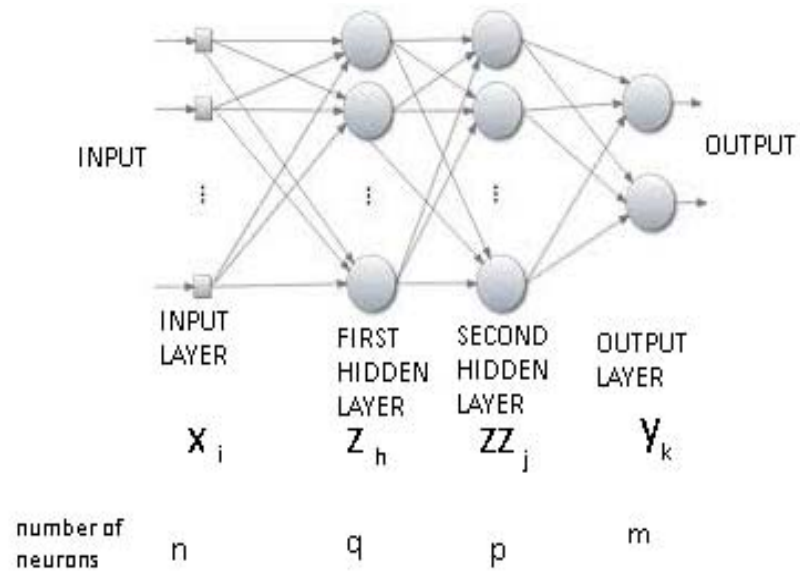

Figure 7. Architecture of neural network

During first phase of feed forward, each input unit $\left(\mathrm{x}_{\mathrm{i}}\right)$ receives the input signal and feeds to all neurons in the next hidden layer through weight matrix ' $U$ ' and ' $\mathrm{u}_{\mathrm{ih}}$ ' is the weight from $\mathrm{i}^{\text {th }}$ unit of input layer to $h^{\text {th }}$ neuron of first hidden layer. Total input for neuron $Z_{h}$ is calculated as

$$
z_{h_{-} \text {in }}=\sum_{i=1}^{n} u_{i h} \cdot x_{i}+u_{0 h} \quad \text { for all h (1 to q) }
$$

Then activations ' $\mathrm{z}_{\mathrm{h}}$ ' of first hidden layer are calculated using its activation function. Similarly these activations are fed to next hidden layer through weight matrix ' $\mathrm{V}$ ' and ' $\mathrm{v}_{\mathrm{hj}}$ ' is the weight from $\mathrm{h}^{\text {th }}$ neuron of first hidden layer to $j^{\text {th }}$ neuron of second hidden layer. Activations ' $\mathrm{zz}_{\mathrm{j}}$ ' $(\mathrm{j}=1$ to $\mathrm{p}$ ) are calculated. Finally these activations are fed to output layer through weight matrix ' $W$ ' and ' $W_{j k}$ ' is the weight from $j^{\text {th }}$ neuron of second hidden layer to $\mathrm{k}^{\text {th }}$ neuron of output layer. Then outputs ' $\mathrm{y}_{\mathrm{k}}$ ' $(\mathrm{k}=1$ to $\mathrm{m})$ are computed. Mean square error is calculated using the desired outputs $t_{\mathrm{k}}$ and fed back to the lower layers. Weights are modified according to the gradient descent method.

Mean Square Error is

$$
\begin{aligned}
& E=\sum_{k} \frac{1}{2}\left(t_{k}-y_{k}\right)^{2} \\
& \frac{\partial E}{\partial W_{J k}}=-\left(t_{k}-y_{k}\right) * \frac{\partial y_{k}}{\partial W_{J k}} \\
& =-\left(t_{k}-y_{k}\right) * f^{\prime}\left(y_{k_{-i n}}\right) * \frac{\partial y_{i n_{-} k}}{\partial \omega_{J K}} \\
& =-\left(t_{k}-y_{k}\right) * f^{\prime}\left(y_{k_{-i n}}\right) * \mathrm{Z} Z_{J}
\end{aligned}
$$


Error term for weights ' $\mathrm{w}$ ' is $\delta_{k}$

$$
\delta_{k}=-\left(t_{k}-y_{k}\right) * f^{\prime}\left(y_{k \_ \text {in }}\right)
$$

Change in weights is $\Delta \omega_{j k}=\alpha \delta_{k}\left(Z Z_{J}\right)$

Now for error term and change of weights for ' $v$ '

$$
\begin{aligned}
& \frac{\partial E}{\partial v_{H j}}=-\sum_{k}\left(t_{k}-y_{k}\right) \frac{\partial y_{k}}{\partial v_{H j}} \\
& =-\sum_{k}\left(t_{k}-y_{k}\right) f^{\prime}\left(y_{i n_{-} k}\right) \frac{\partial y_{i n_{-} k}}{\partial V_{I J}} \\
& =-\sum_{k}\left(t_{k}-y_{k}\right) f^{\prime}\left(y_{i n_{-} k}\right) \omega_{J K} \frac{\partial\left(Z Z_{k}\right)}{\partial V_{I J}} \\
& =-\sum_{k} \delta_{k} \omega_{J k} * f^{\prime}\left(Z Z_{h-i n}\right) Z_{h}
\end{aligned}
$$

Error term for weights ' $\mathrm{v}$ is $\delta_{j}$

$$
\delta_{j}=\sum_{k} \delta_{k} \omega_{J k} * f^{\prime}\left(Z Z_{h-i n}\right)
$$

Change in weights is $\Delta v_{h j}=\alpha \delta_{j} Z_{h}$

Now for error term and change of weights for ' $u$ '

$$
\begin{aligned}
& \frac{\partial E}{\partial u_{I h}}=-\sum_{k}\left(t_{k}-y_{k}\right) \frac{\partial y_{k}}{\partial u_{I h}} \\
& =-\sum_{k}\left(t_{k}-y_{k}\right) f^{\prime}\left(y_{i n_{-} k}\right) \frac{\partial y_{i n_{-} k}}{\partial u_{I h}} \\
& -\sum_{k} \delta_{k} \omega_{J k} \frac{\partial f\left(Z Z_{j}\right)}{\partial u_{I H}} \\
& =-\sum_{k} \delta_{k} \omega_{J k} f^{\prime}\left(Z Z_{j-i n}\right)-\frac{\partial\left(Z Z_{j \_i n}\right)}{\partial u_{I H}} \\
& =-\sum_{k} \delta_{k} \omega_{J k} f^{\prime}\left(Z Z_{j-i n}\right) V_{H J} \frac{\partial\left(Z_{h}\right)}{\partial V_{I H}} \\
& =-\sum_{k} \delta_{k} \omega_{J k} f^{\prime}\left(Z Z_{j-i n}\right) V_{H J} f^{\prime}\left(Z_{h-i n}\right) X_{I} \\
& =\sum_{p} \delta_{j} V_{H J} f^{\prime}\left(Z_{h-i n}\right) X_{I}
\end{aligned}
$$

Error term for weights ' $u$ ' is $\delta_{h}$

$$
\delta_{h}=\sum_{p} \delta_{j} V_{H J} f^{\prime}\left(Z_{h-i n}\right)
$$

Change in weights is $\Delta u_{i h}=\alpha \delta_{h} X_{I}$

New weights can be obtained using equations (1), (2) and (3) for all weights:

weight(new) $=$ weight(old) + change in weight 
This mathematical model for backpropagation training algorithm is used for our proposed predictor. To predict the status of the channel is dependent on previous and current sensed observations. A trained artificial neural network (ANN) is embedded in its control unit.

In this work data is considered as past observations per channel i.e. spectrum unit. The slot of eight values of channel status and their vacant time is fed to Backpropagation Neural network which will predict the next status of channel. Status is either 1 or $-1,1$ means the channel is busy or -1 means the channel is vacant. And value of time varies from 1 to 6 for vacant channels only otherwise it is zero. So the network has sixteen neurons at input layer and two neurons at output layer. Best number of hidden layers and neurons are decides using hit and trial method for the best performance. Too small number of neurons in the hidden layers may result in poor accuracy of the results. With too large number of neurons in hidden layer, network will not be able to generalize the efficiency. Once the architecture is decided, it can be used in CUP and performance of network improves significantly.

\section{SIMULATIONS AND RESULTS}

In this section we will discuss the simulations done and analyze the results for accuracy.

Following steps have been followed:

1. Selection of tools to generate the database.

2. Generate the database using Poisson distribution.

3. Selection of tool to simulate neural network.

4. Design the neural network based on number of input, number of outputs.

5. Decide the number of hidden layers and their number of neurons at random.

6. Analyze the result and redesign the network till minimum MSE is achieved.

Simulations have been carried out in Neural Network Tool Box of MATLAB R2010 of Mathworks, Natick, MA, USA. The data has been generated in statistical domain. The stochastic processes, Poison and Pareto random are used to generate PU traffic and free slots for channel respectively. Poisson process is the traditional traffic generation model for circuit-switched data as well as packet data and number of packets per unit time slot follows the poison distribution. But for more realistic model, Pareto distribution can be used as a long-tail traffic model. Training of the neural network is done with stochastic data. Training in neural networks means the nonlinear mapping of input and desired outputs. This is done by adjusting weights so that mean square error is minimum. As number of input units and number of output neurons are fixed. The number of hidden layers and their number of neurons are decided by hit and trial method. Through simulations it has been observed that network with two hidden layers and with 15 neurons in first layer and 10 in second layer performs the best in terms of MSE. Parameters used for simulations are given in Table 1

Table 1. Parameters for simulations

\begin{tabular}{cc}
\hline Net Type & Multilayered feedforward network \\
\hline Training algorithm & Backpropagation \\
Training function & Trainlm \\
Training data set size & 8000slot (1000 data points) \\
validation data set size & 4000slot (500 data points) \\
No. of hidden layers & 2 \\
No. of neurons in first hidden layers & 15 \\
No. of neurons in second hidden layers & 10 \\
Epochs & 1000 \\
Learning rate & .001 \\
\hline
\end{tabular}

Testing and validation are also done using offline data to validate the accuracy of the network. Training of predictor is done with training data set of 8,000 slots i.e. 1000 data points. Here eight slots represent one data point (eight channels in one resource block). This means 1000 previous values of one resource block are fed to neural network for training. The result is shown after one thousand epochs. It has been observed after training the mean square error is extremely low. For clarity status and free time for 300 data points have been plotted in graphs shown in Figure 8 and Figure 9. 


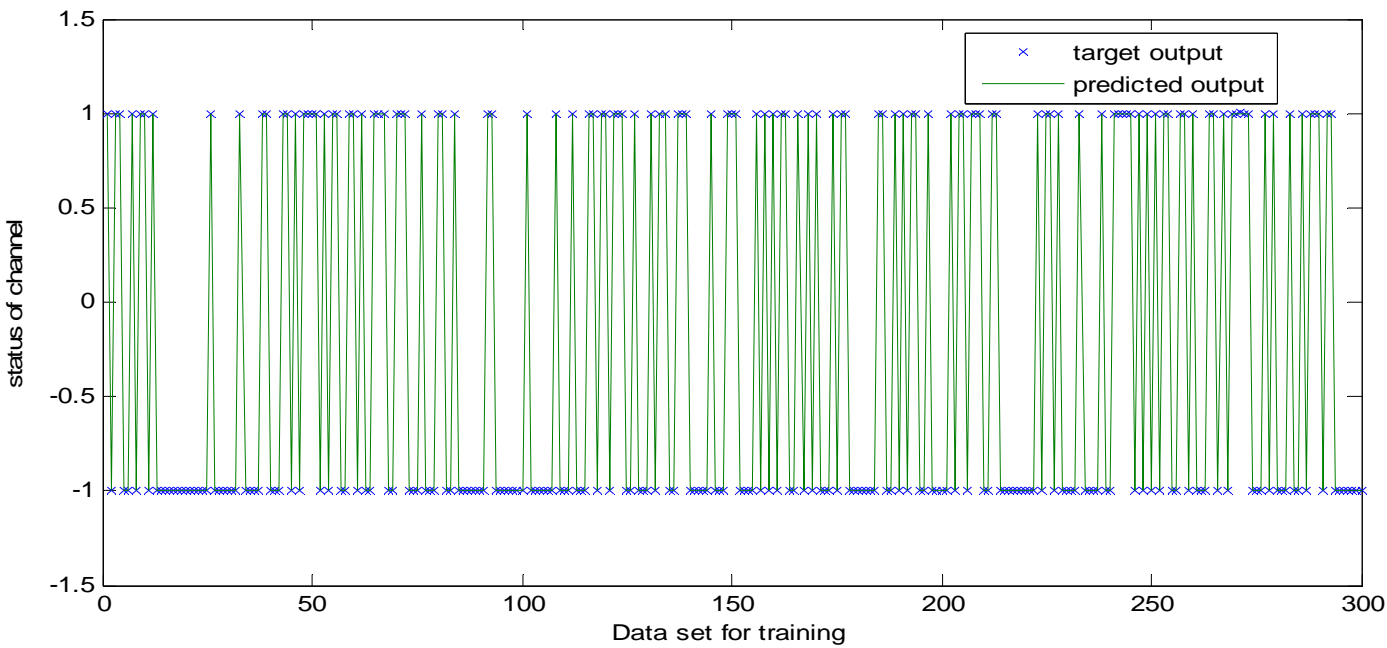

Figure 8. Training of channel status for 300 data points

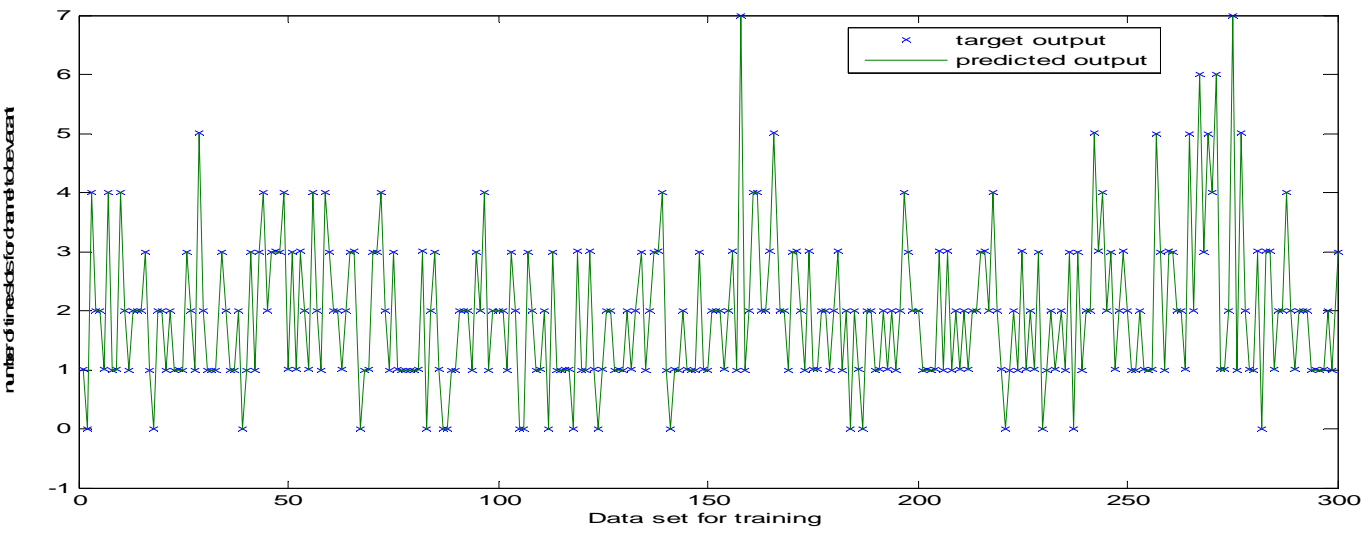

Figure 9. Training of number of time slots for vacant channel for 300 data points

After training and testing of the network we need to apply unseen data to validate it. Validation of predictor is done with training data set of 3200 slots i. e. 400 data points. Results for status of channel and number of idle slots are shown in Figure 10 and Figure 11.

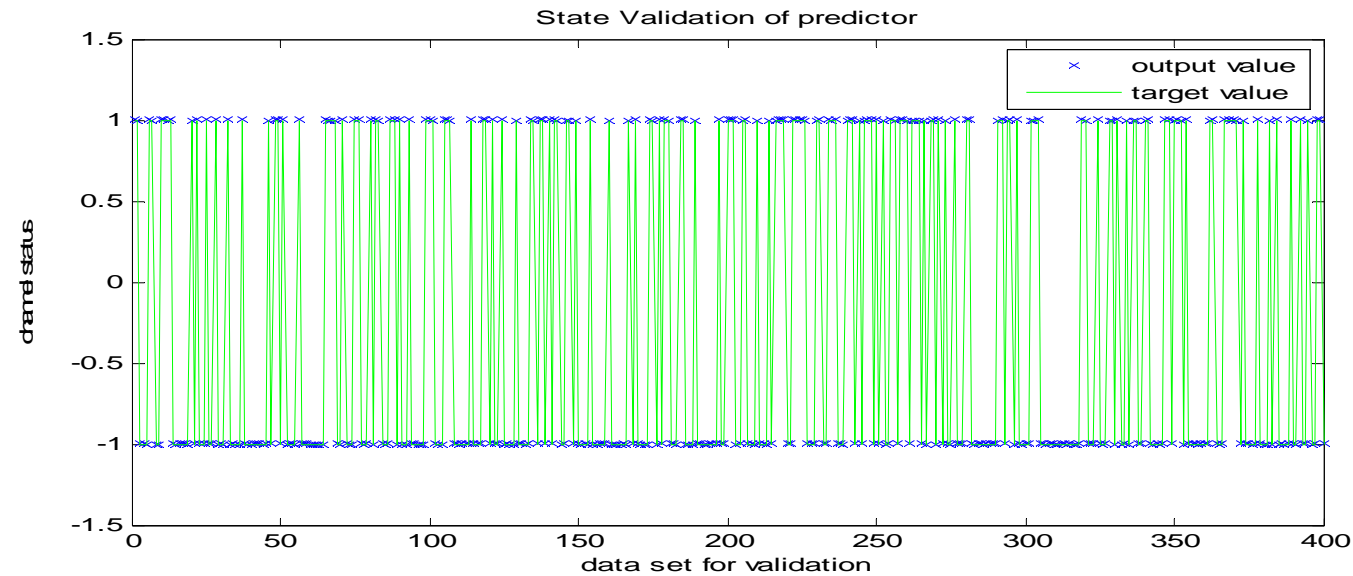

Figure 10. validation of channel status 


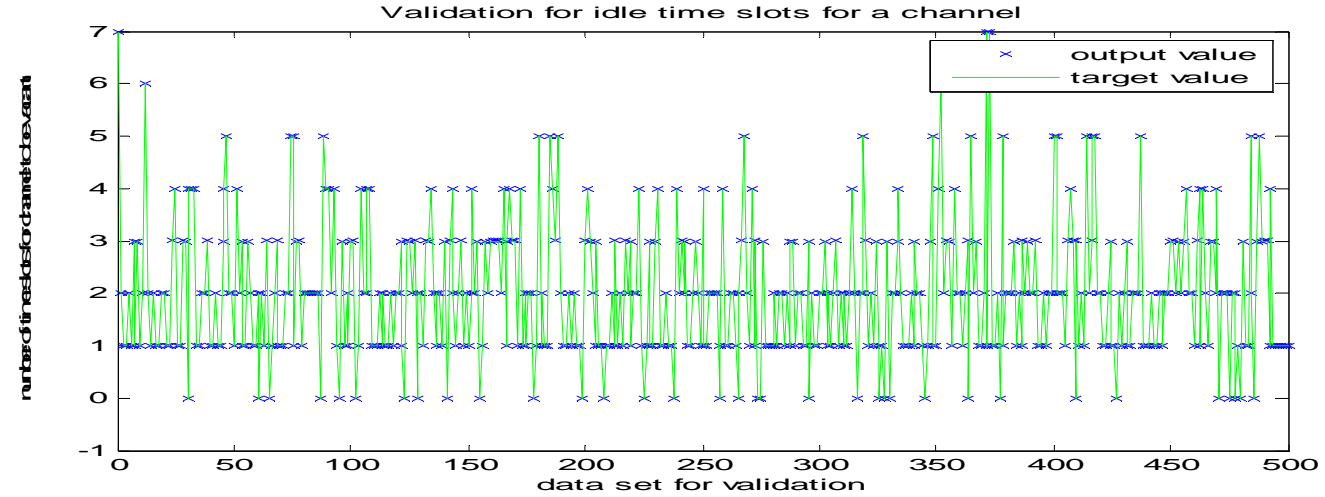

Figure 11. Validation of number of time slots for vacant channel

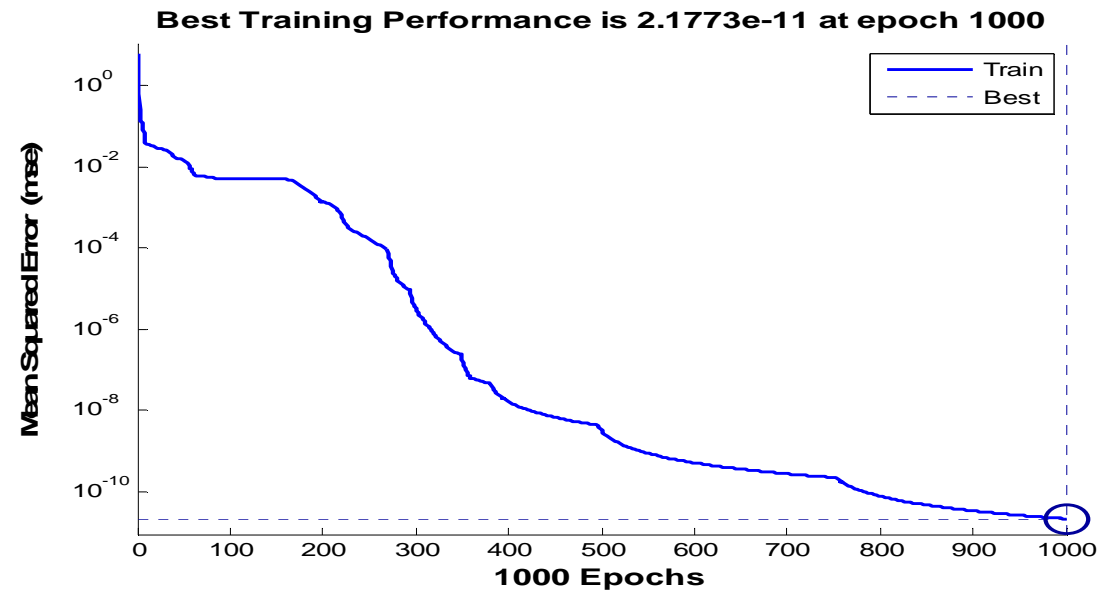

Figure 12. Mean Square error of network

The performance in terms of mean square error (MSE) is shown in Figure 12. It can be observed that after 1000 epoches MSE is of the order of $10^{-12}$. Gradient decent is of the order $10^{-7}$ and zero validation failure. Momentum coefficient (mu) is the fraction of previous weight update to current weight, it's value also very low $\left(10^{-10}\right)$ as shown in Figure 13.
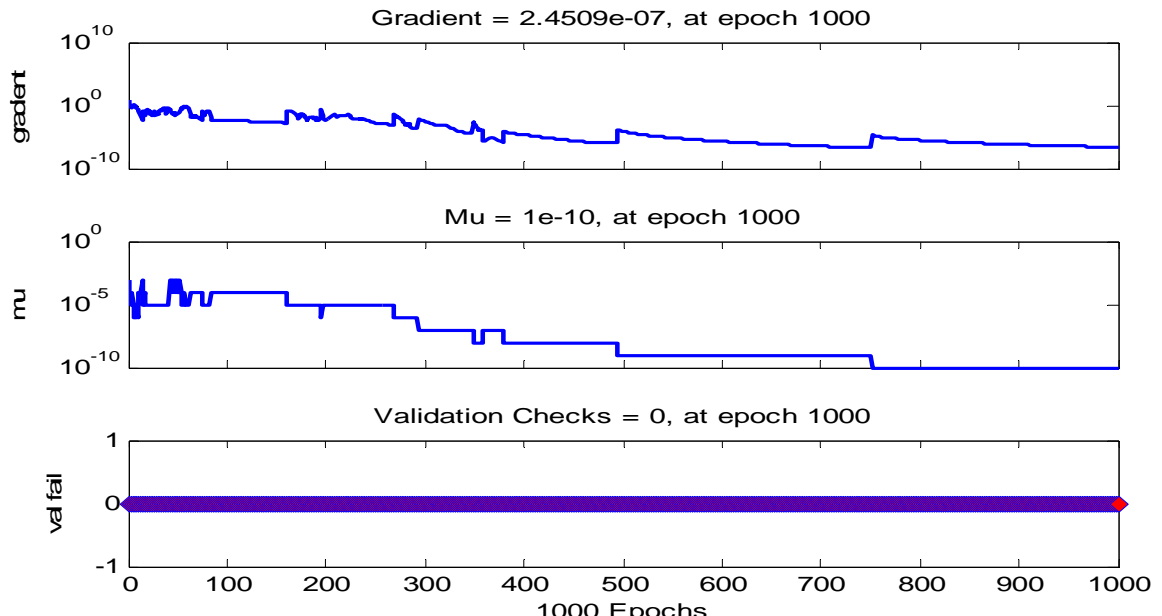

Figure 13. Gradient, mu and validation failure of network during training 
Now the proposed CUP will sense only those channels which are predicted to be vacant for maximum time. This will further minimize the switching over the channels.

\section{CONCLUSION}

In this work problem of future wireless network has been examined and new learning scheme using artificial neural network is proposed. This network has been trained using statistical primary user data for mobile communication and can be embedded in control unit of secondary users. Now secondary user need to sense only those channels which are predicted to be free and select appropriate channel for transmission. This proposed technology will not only save time and energy for spectrum sensing but also improves spectrum utilization. Further once the artificial neural network is trained, its computational complexity is minimum.

\section{REFERENCES}

[1] J. Mitola, “Cognitive Radio: An Integrated Agent Architecture for Software Defined Radio”, Ph.D. thesis, Royal Institute of Technology, 2000.

[2] S. Haykin, "Cognitive radio: Brain-empowered wireless communications”, IEEE Journal on Selected Areas in Communications, Vol. 23, No. 2, pp. 210-230, Feb. 2005.

[3] Maziar Nekovee, "Dynamic spectrum access with cognitive radios: future architectures and research challenges", IEEE 1st International Conference on Cognitive Radio Oriented Wireless Networks and Communications (CROWNCOM), Greece, pp 1-5, June 8-10, 2006.

[4] Ashish Bagwari , Geetam Singh Tomar, "Adaptive double-threshold based energy detector for spectrum sensing in cognitive radio networks”, International Journal of Electronics Letters, Vol. 1, Issue 1, pp 24-32, 2013

[5] Tsung-Hui Chuang, Guan-Hong Chen, Meng-Hsun Tsai, Chun-Lung Lin, "Alleviating Interference through Cognitive Radio for LTE-Advanced Network", International Journal of Electrical and Computer Engineering (IJECE) Vol. 5, No. 3, pp. 539-547, June 2015

[6] Gamal Abdel Fadeel Khalaf, "An Optimal Sinsing Algorithm for Multiband Cognitive Radio Network", International Journal of Information \& Network Security (IJINS) Vol. 2, No. 1, pp. 60-67, February 2013

[7] Mohammadreza Amini , Asra Mirzavandi , "Phase-Type Model Spectrum Sensing for Cognitive Radios”, IETE Journal of Research, published online 25 ${ }^{\text {th }}$ Mar 2015, pp 1-7.March 2015.

[8] Tsagkaris K, Katidiotis A, Demestichas P., "Neural network-based learning schemes for cognitive radio systems", Computer Communications, 2008; 31(14), pp 3394-3404, Vol 31, 2008

[9] M. Bkassiny, Y. Li, S.K. Jayaweera, "A survey on machine learning techniques in cognitive radios", IEEE Communications Surveys \& Tutorials, vol. 15, no. 3, pp. 1136-1159, March. 2013.

[10] VK Tumuluru, W Ping, D Niyato, “A neural network based spectrum prediction scheme for cognitive radio”, IEEE International Conference on Communications (IEEE ICC), 2010, Cape Town, pp. 1-5. 23-27 May 2010.

[11] Zhenyu Zhang, and Xiaoyao Xie, "Intelligent cognitive radio: research on learning and evaluation of CR based on neural network”, 5th International Conference on Information and Communications Technology, ICICT 2007, Cairo, pp 33-37, 16-18 Dec. 2007.

[12] MI Taj, M Akil, "Cognitive radio spectrum evolution prediction using artificial neural network based multivariate time series modeling”, 11th European Wireless Conference 2011 - Sustainable Wireless Technologies (European Wireless), (Vienna), pp. 1-6. 27-29 April 2011

[13] A. He , K.K. Bae, T.R. Newman, J. Gaeddert, K. Kim, R. Menon, L. Morales-Tirado, J.J. Neel, Y. Zhao, J.H. Reed and W.H. Tranter, "A survey of artificial intelligence for cognitive radios", IEEE Transaction on Vehicular Technology, Vol. 59, No. 4, pp.1578 -1592, 2010

[14] JD Deaton, RE Irwin, LA DaSilva, "The effect of a dynamic spectrum access overlay in LTE-Advanced networks", IEEE Symposium on New Frontiers in Dynamic Spectrum Access Networks (DySPAN), Aachen, pp. 488-497. 3-6 May 2011

[15] O Vucenta, H Carlos, JF Monserrat, G Xavier, “Implementing opportunistic spectrum access in LTE-Advanced”, EURASIP Journal of Wireless Communication and Networking, Vol. 99, 2012

[16] 3GPP TS 36133, Evolved Universal Terrestrial Radio Access (E-UTRA); requirements for support of radio resource management, April 2010. http://www.etsi.org/deliver/etsi_ts/136100_136199/136133/9.03.00_60/ts_13 $6133 v 090300 p . p d f$

[17] N. Baldo and M. Zorzi, "Learning and Adaptation in Cognitive Radios using Neural Networks", 5th IEEE Consumer Communications and Networking Conference (CCNC 2008), pp. 998-1003, January 2008

[18] Z. Zhang and X. Xie, "Intelligent Cognitive Radio: Research on Learning and Evaluation of CR Based on Neural Network", Proceedings ITI 5th International Conference on Information and Communications Technology (ICICT 2007), pp. 33-37, December 2007.

[19] C.J. Rieser, T.W. Rondeau, C.W. Bostian, and T.M. Gallagher. "Cognitive RadioTest bed: Further Details and Testing of a Distributed Genetic Algorithm Based Cognitive Engine for Programmable Radios”, IEEE Military Communications Conference, MILCOM, October 2004.

[20] X. Zhu, Y. Liu, W. Weng, and D. Yuan, "Channel Sensing Algorithm based on Neural Network for Cognitive Wireless Mesh Network”, Proceedings of IEEE International Conference on Wireless Communications (WiCom), pp. 1-4, 2008. 


\section{BIOGRAPHIES OF AUTHORS}

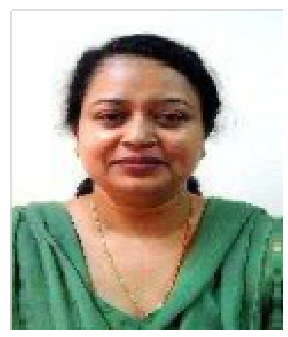

Rita Mahajan was born in India, Punjab, in 1965. She did her B.E. (E\&EC) Degree from Thapar University, Patiala in 1986 and her M.E. (Electronics) in 1993 from Punjab Engineering College Chandigarh, India. She has got 25 years of teaching experience. She has published more than 20 papers in International and National journals and conferences. Her research interests include neural networks and cognitive radios.

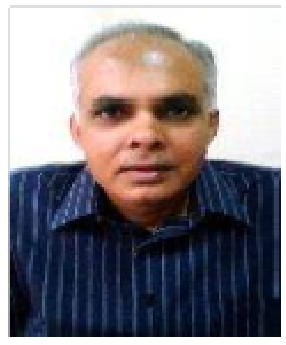

Deepak Bagai was born in India, Chandigarh, in 1963. He did his B.E. (E\&EC), M.E. (Electronics) and Ph.D from Punjab Engineering College Chandigarh, India in 1985, 1992, and 1997 respectively. He has got 28 years of teaching experience. He has published more than 40 papers in International and National journals and conferences. His research interests include telecommunications and wireless communications. 